\title{
Von Brecht lernen
}

$\mathrm{D}$ er alte Augsburger ist heute immer noch ein Quell der Erkenntnis. Eine im „Leben des Galilei“ geschilderte Szene könnte dem heutigen Medizinbetrieb entstammen: Galilei will der Kurie beweisen, dass sich die Erde um die Sonne dreht. Dazu hat er ein Fernrohr mitgebracht, durch das die hohen Kardinäle und Exzellenzen hindurchschauen sollen, um sich von etwas zu überzeugen, das nach den Lehren der Kirche nicht existieren dürfte: ein zusätzlicher Jupitermond. Doch anstatt den Tatsachen ins Auge zu blicken, ergehen sich die Kirchenfürsten in langen theoretischen Diskursen, warum das Offensichtliche theoretisch nicht möglich ist. Damit lassen sie den Forscher stehen - ohne sich in die Gefahren der Realität zu begeben. Galilei ruft ihnen hinterher: „Aber die Herren brauchten wirklich nur durch mein Instrument zu schauen!“

Von der Kurie zur Klinik ist es kein großer Schritt. Unbeirrbar fest glauben wir an die radikale Prostatektomie als die allein selig machende Behandlung des Prostatakarzinoms niedrigen Risikos. Tausend Gründe werden von der „Kurie“ angeführt, um therapieren zu können. Sie alle werden hergeleitet aus zweitklassigen Daten, Überzeugungen und der eigenen Erfahrung. Unsere Wahrnehmung ist die der damaligen Kardinäle. Die sahen mit eigenen Augen, dass sich die Sonne um die Erde drehte. Wir sehen all die Männer, die wir operieren und dann geheilt nach Hause entlassen. Dass die Operation nicht Ursache für das gute Ergebnis ist, diese Deutung lässt unser Glaube nicht zu. So verweigern wir uns dem, was unser Weltbild ins Wanken geraten lässt:

Zwei randomisierte kontrollierte Studien zeigen einen erschreckend geringen Nutzen der RP gegenüber Nichtstun. Bei Bill-Axelson et al. haben nach 15 Jahren sechs von hundert operierten Männern aller Risikogruppen von dem Eingriff profitiert. Im Umkehrschluss wurden 94 von hundert Männern umsonst operiert, das heißt sie mussten überflüssigerweise eine Körperverletzung hinnehmen. Die PIVOT-Studie verdeutlicht diesen Trend: Patienten mit Tumoren der niedrigen und mittleren Risikogruppe hatten keinen Nutzen von der Operation - wiederum im Vergleich zu Nichtstun.

Für niedriges Risiko empfiehlt die S3-Leitlinie unter anderem die aktive Überwachung (AS) mit verzögerter Intervention bei Anzeichen eines Progresses. Gegen diese
Strategie wird mit liturgischer Gleichförmigkeit eingewandt, es sei eine experimentelle Strategie, weil Langzeitdaten fehlten und keine vergleichenden Studien vorlägen. AS sei gefährlich, weil man den rechtzeitigen Zeitpunkt zur Heilbehandlung verpassen könne. Und auf den ersten Blick scheinen diese Argumente Tatsachen zu beschreiben, so wie das Bild der um die Erde wandernden Sonne aus der bloßen Anschauung wie eine Tatsache erscheint, wenn man nicht über die nötigen astronomischen Einsichten verfügt. Deshalb ruft die Kurie nach neuen Markern, bunten Bildern und randomisierten sinnleeren Studien bis 2023.

Wagt man den Blick durch das Fernrohr, stellen sich die Tatsachen jedoch anders dar: Die Logik sagt, dass eine verzögerte Intervention (AS) hinsichtlich des Nutzens keine schlechteren Ergebnisse bringen kann als keine. Für keine Intervention versus radikale Prostatektomie (RP) liegen Ergebnisse aus zwei randomisierten kontrollierten Studien vor (siehe oben). Die eine weist keinen Nutzen für Männer über 65 nach und die andere keinen Nutzen für niedriges und mittleres Risiko. Daher kann also eine verzögerte Intervention bei Männern über 65 oder bei geringem und mittlerem Risiko keinen Nachteil gegenüber der RP haben, wenn dort schon das Unterlassen jeglicher Intervention nicht zu schlechteren Ergebnissen führt - logisch. Zugegeben, die Studienlage für AS selbst entspricht nicht der höchsten Evidenz. Es ist aber - wie oben nachzulesen - keine intellektuelle Herausforderung, die indirekte Evidenz höchsten Grades dafür herzuleiten. Der Blick durch das Fernrohr aber erfordert Mut, weil man sich den Konsequenzen des Erkannten stellen muss. Brechts Kardinälen fehlte dieser Mut und sie retteten ihre Ideologie durch Verweigerung und das Herbeten veralteter Litaneien. Die Wissenschaft und die Geschichte haben ihnen Unrecht gegeben. Vielleicht bringt der eine oder andere „Kardinal“ diesen Mut in der nächsten Zeit auf. Die vielen Männer, die wir vor den Folgen einer sinnlosen Operation bewahren können, werden es danken.

Ihr

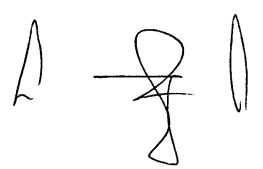

